of zine sulphate the inflammation subsided in a few weeks. For the purpose of determining to what extent the germ was to be found in Philadelphia, he made spreads and cultures of the conjunctival discharge in 31 additional cases of conjunctival and corneal disease seen in the Jefferson and Polyclinic hospitals, and found the diplococcus present in 6 cases. The patients examined included those suffering from phlyctenular disease, hypopyon keratitis, trachoma and simple corneal ulcers. In two of the eases showing the germ there were small ulcers of the cornea.

The results of this study were presented to the Section on Ophthalmology of the College of Physicians of Philadelphia on Dee. 20,1898 , and the abstract of the paper printed in the proceedings of the college, but the original paper was not published, as he intended carrying the investigations further, but failed to do so. At that meeting he exhibited a shoemaker with ehronic inflammation of the conjunctiva of both cyes, which had existed for a long time. There was a moderate secretion, the veins in the fornix were prominent and the vessels on the globe were slightly congested and tortuous. Examination of the scanty discharge showed the diplobacillus. For the purpose of determining the effect of the remedies ordinarily employed for ehronic conjunctival inflammation the man was given treatment at the dispensary for several weeks with tannin, silver, boroglycerid and weak bichlorid of mercury, and at home a saturated solution of boracic acid. No improvement could be detected, so that a solution of zine sulphate, I grain to the ounce, was ordered, and in about a week the inflammation had subsided sufficiently to relieve the patient of the annoying symptoms of which he had complained. From a study of the well-developed germ there is seen to be a clear space surrounding most of the double rods, indicating some form of envelope, and Dr. Sweet therefore felt at that time, as he does now, that the contention made by Gifford that the germ had a eapsule was correct, although denied by Morax, Axenfeld and Peters.

Dr. Citarles H. Wrlltams, Boston, mentioned a practical point in regard to the use of zine. It will often be found beneficial to use the sozoiodolate of zinc in the same strength as the solution of sulphate, especially if combined with chloretone (a solution of chloretone 1 grain to the ounce). The chloretone preserves the solution and acts as a sedative to the conjunctiva, so that the applications are less irritating.

Dr. Nelson M. BLACK, Milwaukee, declared that he has noticed that in these cases of diplobacillus conjunctivitis the patients almost invariably complain of symptoms closely allied to those due to refractive errors and report that they have had their glasses changed a number of times without benefit. This shows the importance of a bacteriologic examination of the contents of the conjunctival sac in many refractive cases.

Dr. E. V. L. Brown, Chicago, spoke of a case of Bacillus subtilis infection following a penetrating wound of the sclera. The foreign body was removed with the magnet and panophthalmitis developed. The eye had to be eviscerated and a pure culture of the Bacillus subtilis was obtained. The cccurrence of the Bacillus subtilis is infrequent, but he thinks it is significant as showing that the intraocular structures are susceptible to such organisms. Roemer has contended that in sympathetic ophthalmia the eye is susceptible to certain organisms that do not affect other parts of the body.

Dr. Brown Puser, Chieago, said that he has some histologic sections which are most instructive. He had these with him and had hoped to show them. The sections are from the famous and unique case of Stock, and he is indebted to him and to Professor Axenfeld for them. If an ophthalmologist sees these sections and the upset condition of the conjunctiva he will realize that it is not remarkable that zinc does not effect a rapid cure. The failures spoken of from this remedy, Dr. Pusey thinks, are due to the fact that a rapid cure is expected and the treatment is not persisted in. In this form of conjunctivitis long and persistent treatment is necessary.

Dr. Edward A. Shumwax, Philadelphia, said in regard to the capsule to which Dr. Sweet has referred that, while it is known that Gifford contended that it was present, Axenfeld rontended that it was not, but in his last article Axenfeld concludes that although it is not demonstrable by ordinary stain- ing methods it is probably present and may be shown by the aid of special staining methods.

Dr. John E. Weers, New York, said that the Bacillus xerosis is not considered pathogenic by those who have studied it. Dr. Weeks syoke of a case he followed up, in which the disease occurred in in infant of a few months. The child died and an autopsy was held. The disease presented itself as one of xerosis of the conjunctiva. The membrane was found on the palpebral and ocular conjunctiva. All the solid organs of the body were examined. From the pelvis of the kidney the bacillus was recovered and the characteristic deposit was found there. In the substance of the kidney the micro-organism was also found. From the liver also it was recovered. The bacillus was found in practically all the tissues of this infant. The child died apparently of marasmus, but it seems to Dr. Weeks very peculiar that this general infection should be found unless there is some pathogenic quality possessed by this bacillus. The bacillus has also been recovered from the urine in cases of xerosis conjunctivitis that wore not fatal.

In reference to Dr. Shumway's statement that the staplylococcus has no causative relation to phlyctenular conjunctivitis, Dr. Weeks said that some years ago he made a large number of examinations of the nodes or elevations found in phlyctenular conjunctivitis, and he found that by removing the contents of these elevations carefully and aseptically pure staphylococcus cultivation was obtained almost invariably. This also occurs in the little nodules of moist eczema. Some few experiments have shown that small nodules can be produced by the introduction of the staphylococcus into the mucous membrane of the lids and also into the skin of the arm. All physicians know that in order to develop the phlyctenular process a suitable condition of the system must exist, but this suitable condition, it seems to Dr. Weeks, may exist, and in the light of these experiments permit the staphylococcus to be the exciting cause of phlyctenular trouble. It is his opinion, he said, that the phlyctenular process that occurs in connection with acute contagious conjunctivitis is due to the Staphylococcus pyogenes aureus, the tissue having been prepared for invasion by the local condition. It has been remarked in connection with the ulcers of the cornea produced by the diplobacillus that the staphylococcus was found. The reason is that these ulcers, originating from the diplobacillus, are infected by other organisms and the destruction of tissue is not due to the action of the diplobacillus alone, but the other micro-organisms as well.

In regard to the Bacillus subtilis, cases of such infection have been reported following injury, but none of them have been due to infection, without traumatism. The Bacillus sub. tilis is not infrequently recovered from the normal conjunctiva and Dr. Weeks has reported cases of this kind.

\section{THE RELATION OF SYSTEMIC DISEASES TO THE CONDITIONS OF THE ORAL CAVITY.*}

\section{JAMES EDWARD POWER, D.M.D. PROVIDENCE, R. I.}

The importance of the study of the pathology of the oral cavity in connection with the treatment of systemic diseases is obvious, and he who is ignorant of its teachings is not only unjust to his patients, but must be regarded as a hindrance to the progress of his profession.

We often hear the study of micro-organisms referred to as the new or germ theory, and, unfortunately, not only by those who deal in glittering platitudes of which they are ignorant, but often by members of our own profession who make statements which cause us to feel that this most important part of their technical education has been neglected. The great Roman author, Varro, in his writings during the first century before Christ, called

* Read in the Section on Stomatologr of the American Medical Association, at the Fifty-seventh Annual Session, June, 1906. 
the attention of the world to the fact that some indefinable, invisible, living organisms were responsible for certain diseases and especially for those of an epidemic character. During the sixteenth century Leewenhoek was able to observe, through the magnifying lens of the microscope, definite outlines of these micro-organisms, and he gives a classification, the first and most important step in the scientific investigation of disease. The forms which this great scientist discovered at that time do not correspond to the forms which we find to-day. The whole world is glar! to believe, however, that this error, if it may be called one, was due to the instrument and not to the man. Every effort that is being made to draw aside the curtains and to reveal the life history of these micro-organisms allows the light of science to shine more brightly and to illume the background whereon is written the name of this Swiss naturalist, Leewenhoek, the first investigator, whose successors are Pasteur, Raynaud, Tannelongue, Vulpian, Moriggia, Marchiafava. Sternberg, Klein, Miller and many others.

TILE MOUTH AS AN INCUBATOR.

We can easily understand if we familiarize ourselves with the fundamental principles of bacteriology that the mouth is an excellent incubator for the development of micro-organisms, principally because it contains heat, moisture and oxygen, the conditions on which they thrive. It is true that many bacteria can not live in the mouth on account of the variations of these conditions, but it is equally true that nearly all the pathogenic or death-producing micro-organisms find in the mouth an environment. which is perfectly suited for their habitation. Miller has isolated more than 100 different specimens from the mouth.

ANTISEPTIC PROPETTIES OF TIIE SALIVA.

Many are under the impression that the saliva contains antiseptic properties and base their conclusions on the well-known fact that the mouth does not yield rery readily to the ravages of infection. According to Miller and other investigators, the antiseptic properties of the saliva are so slight that they should not be considered in dealing with this problem. The reason that infection does not take place more frequently is that the mouth and its component parts have greater power of resistance than other portions of the body. 'The fact that so many kinds of bacteria do exist in the oral cavity is sufficient to satisfy the average student that the saliva does not contain the antiseptic properties which some are inclined to believe that it does. If this were so, then the bacteria would be destroyed.

MICRO-ORGANISMS FOUND IN TILE MOUTH.

In neglected mouths $1,140,000,000$ cultivable microorganisms are found. The accumulation which is found between the teeth, on the shelves made by overhanging fillings, in the cavities of carious tecth, in the pockets made by malposed teeth, etc., is so mixed that it makes a most excellent medium for the growth of these microorganisms. Even in the average mouth each drop of saliva contains 4,375 micro-organisms, among them being streptococci, staphylococci and diplococei. Flïgge has demonstrated by his experiements that any explosive action of the lips, such as the pronunciation of words commencing with $\mathrm{D}, \mathrm{K}, \mathrm{P}$ and $\mathrm{T}$, will expel these virulent bacteria into the air. This is sufficient evidence, or at least should be, to make us pause a moment and ask ourselves whether or not the mouths of the children in our public schools should be subjected to frequent examinations.
It is impossible for me in this brief time to point out the far-reaching influence which these little messengers of disease and death can have whose home is the oral cavity. Not only are the antiseptic properties of the saliva of the individual overestimated, but the gastric juice is also regarded as the destroyer of any germs that may be swallowed. But MacFadyan, Sucksdorf, Miller, Baumgarten and others have demonstrated that the action of the gastric juices does not destroy the bacteria found in the mouth. Sucksdorf found an average of 380,000 cultivable bacteria in one milligram of fresh feces and that 30 per cent. of these bacteria were carried from the mouth to the anus.

According to the authorities, the only time that the gastric juice has the power of destroying bacteria is when digestion is most active, two or three hours after a meal. This, one can readily understand, is the time when these micro-organisms from the oral cavity are least apt to be introduced, as most, if not all, the pathogenic organisms are carried in by the food when it passes through the mouth. Hence. it would be folly to argue that this is not also a great danger.

Of the many specimens of bacteria found in the mouth perhaps the most important are those of pathogenic type, such as diphtheria, typhoid, cholera, septicemia, pneumonia and others whose development and propagation cause in many instances a cessation of life.

Netter found that 10 per cent. of all mouths examined by him contained the Staphylococcus pyogenes aureus and Staphylococcus pyogenes albus and that the microorganisms of pneumonia were present in 15 per cent. of healthy mouths. Schriever tells us that 75 per cent. of apical abscesses examined by him contained the microorganisms of pneumonia, and Vulpian produced septicemia by inoculating animals with the saliva from healthy men.

From the observations and conclusions drawn from the study of discases in our hospitals it has been proved that these micro-organisms are the principal cause in nearly every disease known to modern medicine. The pathologic manifestations vary, however, and in some instances we may find that the inflammatory process involves only the first or hyperemic stage, and in others we find manifestations of the last, or suppurative stage. In the oral cavity under these conditions we find caries and necrosis of both the osseous and muscular tissues, while in other cases the kidneys, lungs, liver, intestines, and the valves of the heart, through the lymphatic glands become involved, and in other instances and by other avenues the brain becomes involved. Indeed, it is beyond the capacity of the human mind to determine just when these minute forms of life cease to invade, cease to change physiologic to pathologic conditions, health to disease, life to death.

According to credible authority, septicemia and pyemia cause the greatest number of deaths, secondary meningitis holds the second place, having been occasioned by the inflammatory process reaching the covering of the brain (pia mater).

When we consider these statements, which are all based on fact, and when we reflect on the conditions which are so favorable to the transmigration of bacteria, such as the highly vascular tissues, the saliva, the glands, etc., with which the mouth is so abundantly supplied, does it not seem strange that more attention is not given to this part of our economy as an etiologic factor in disease? Science has proved their existence; therefore, it 
devolics on us to take measures not only to destroy them, but to destroy all conditions which are conducive to their growth.

\section{INFLAMAATIONS DLRING THE FIRST DENTITION.}

Perhaps the first and most common form of inflammation with which we have to deal is that which presents itself' during the first dentition of the child. 'This is the result of pressure on the tissues caused by the tooth in trying to pass through the gum during the process of eruption. If you pause for one moment you can easily understand the processes which are taking place in the living tissues and which cause so much heat and pain. 1 have known of careless people who had charge of babies introducing all sorts of unclean substances into the mouths of these little sufferers during this periol. It is needless to describe the effect the inflammatory condition has on the nervous system. Suffice to say that all sorts of pathologic reactions in this system, including high temperature, diarrhea, and in many cases convulsions occur. Children have come under my observation suffering from the above described conditions, after having been treated by physicians with internal remedies. which in some instances afforded temporary relief, but in all cases a reaction followed which lessened the child's resisting capacity. A crucial incision through the tissues in the area through which the tooth was trying to pass caused permanent relief. A complete cure of a large number of convulsing children during the period of dentition, and especially during the summer months, causes me to bring this experience before this Scetion for discussion.

When we consider the number of unclean substances, such as soiled fingers. soiled linen, soiled hard-rubber rings, and unclean soft-rubber nipples which are used on nursing bottles, we have ample reason to marvel that the mortality and sick list among these children are not greater. Along pathologic lines, it seems reasonable to assign many of the cases of inflammatory conditions of mothers breasts to transmission by the infant.

\section{TRANSMISSION OF DISEASE BY MOUTII.}

Now, transmission of disease by the mouth is encouraged to a large degree by the aduit, and in the majority of cases it is due to ignorance; for example, in the common practice of moistening promiscuously the lead in pencils, without having previously known whether they were charged with specific bacteria or not. In other instances. infection may take place when the subject may be innocent.

Miller reports an instance where several boys were inoculated with syphilis, after having been tattooed by a syphilitic, who moistened the needle used for the purpose before breaking the skin of the victim.

In one of the Russian provinces all the natives believe that any and all inflammatory conditions of the eve are due to the lodgment of foreign bodies, and the methol alopted is to place the tongue between the ljds of the ere to remove it. Some have become so skilled in this line, that they make a specialty of treating eyes in this way. Acting in this capreity one woman inoculated 6.5 per cent. of the entire population of the province of Wiatka with syphilis.

\section{BACTERTA OF THE MOUTH.}

Netter, Schriever and other scientific investigators have demonstrated that the dental pulp is a source of almost unlimited infection of the vital parts of our svstem. For instarice, as a sequel of this condition we ob- serve necrosis, diseases of the sinuses, infection of the glands of the neck, gastric disturbances and many others which time will not permit me to mention.

Miller found organisms of pyorrhea alveolaris which he called Micrococei gingive pyogenes, virulent in character. Injections through the skin of mice, caused inflammation of the part followed by an abscess which finally resulted in the death of the animal. Introduction of this organism into the abdomen of the mice causes death from peritonitis within 24 hours, the autopsy showing large numbers in the abdominal cavity.

Bacterium gingiva pyogenes is another form of microorganism which is generally found in the mouth of a person affected with pyorrhea, as well as in the substances of a suppurating tooth pulp, and when injecter into the ablomen of white mice will cause death in from 10 to 24 hours. 'The animals' eyes seemed glued together and the spinal column was bent almost like a bow, resembling the conditions of strychnin poisoning. Autopsy showed death from peritonitis.

Bacillus pulpoc pyogenes is still another form of animal life which is found in putrescent pulp, and which when injected into the abdominal cavity of white mice produces death in from 18 to 30 hours.

Another organism found on the superficial lavers of carjous dentine, called Bacillus dentatis viridans, when injected into the skin causes severe inflammation and in one case death from blood poisoning. When injected into the abdomen of white mice death from peritonitis ensued in 60 per cent. of the cases in from 22 hours to 6 days. In these cases, the organisms could not be found in the bloor microscopically, but cultures made from the blood of the hoart developed the bacteria injected.

I call your attention to the action of the micro-organisms, not to have you believe that they will produce like results in the human system, but that their action is the same, only the great resistance of our systems compared with that of mice prevents death, but the resistance is not sufficiently able to prevent an interference with the functions of the organs, therefore we have diseases of other parts. which we can reasonably attribute to the presence of these organisms in the oral cavity.

DEATHS FOLLOWING INFECTIONS OF THE MOUTH.

During the sixtecnth century Vulpius in his writings states that disfiguration and death are the result of toothache, and reports the death of Gosvin Halt, the Amsterdam plysician, who had his gum lanced to allow the eruption of a molar tooth. Subsequently the gum became swollen, insomnia and delirium followed, and finally death reliered him of his sufferings. This condition is what we would probably call to-day septicemia. due either to the micro-organisms being introduced into the sistem from the unclean instruments, or that the instruments made an avenue through which the microorganisms, already present in the mouth, entered the system.

Zakhaveritsch reports the death of two healthy physicians, one from ostcomyelitis. six days after the extraction of a left inferior second molar and another from peritonitis and osteitis. The first stage in the process of each disease was also conclusive evidence that infection was caused by unclean instruments. Banner reports many cases among them being one of especial interest. that of a young student, 24 years old, who made a visit to a phrsician for the purpose of having a molar tontle extracterl. The phrsician broke the tooth in the attempt and luring the next $2+$ hours, swelling ensued. 
Finally the periostium became involved, and necrosis followed. The necrotic area was removed 14 days after; swelling continued, there were chills, great debility. rapid and marked excursions of temperature, pyemia, and finally the right lung became involved with pus, the patient died.

Delester reports several cases in which the inflammatory process spread to the brain and the patient died from menigitis.

The following case reports may be of some interest:

CASE 1.-On Dec. 4, 1905, C. N., a girl, aged 7 , was referred to me by her physician. She was suffering from neuralgia involving the right side of the head, inability to sleep, loss of appetite, etc.

History.--On Nov. 30, 1905, she came home from school complaining of headache, with slight swelling of the right side of the face. Dr. $H$. was called and found the child with a temperature of $105^{\circ} \mathrm{F}$. and other symptoms which would suggest that she was in the first stages of some one of the exanthematous diseases. He prescribed, and visited the child on the next day, expecting to find his diagnosis verified, but on the contrary the swelling had increased somewhat, with a drop of 2 degrees in the temperature. Examination of the throat revealed nothing to the experienced eye of this physician. He continued to visit the child for the next five days, keeping her as comfortable as possible, but was unable to diminish the temperature more than 1 degree. Temperature remained 102 degrees during this time.

Examination.-When I saw her she had a temperature of $102^{\circ} \mathbf{F}$., with the other symptoms previously described. Her mouth seemed to be in a healthy condition, with the exception of two or three badly decayed teeth, scattered about the mouth. The child could not give the exact location of pain. On the superior right maxilla all the teeth were present, including a newly erupted sixth year molar, which was apparently healthy so far as visual and instrumental examination could determine. Anterior to this was the second temporary molar, decayed, although the decay did not involve the pulp. The condition was such as one generally sees in the mouths of children when the decay seemed to have stopped, not unlike the teeth of tobaceo chewers. There was no pain associated with the examination of the decayed tooth, not even on pressure. There was, however, slight pain, when percussion was used on the permanent molar.

Treatment.-I finally decided to extract the permanent tooth, to the great disappointment of the parents, who thought that if any tooth should be extracted it should be the decaved one. Examination of the permanent molar showed unmistakable signs that my diagnosis was correct. Abscesses about the size of small peas were found at the ends of the roots. All other parts of the tooth were normal, having no cavity, not even the small fissure cavity that is present in nearly all first permanent molars. The child made a rapid recovery; her temperature became normal within twenty-four hours.

Since physicians are the ones who are generally called to see such cases, I am going to report one of many cases which I hope will show how important are the lower third molars, or what are commonly called the wisdom teeth, as factors in causing disease.

CAse 2.-On April 9, 1906, L. M. manifested the following symptoms: Severe neuralgia pains, loss of appetite, sleeplessness caused by the pain, finally swelling of the right side of the face, sore throat, and interference with the motion of the jaw, followed by fever and chills. On the second day a physician was called who evidently decided that the patient had some tonsillar infections. He increased the man's suffering by making an incision through one of the tonsils. After three days the patient discharged this physician, and called in another who found him with the described conditions increased and a temperature of 104. He immediately had the man transferred to a private hospital, where, on examination, he found an inferior third molar badly decayed, which he immediately extracted without breaking, followed by a large amount of pus. The patient experienced relief, and temperature dropped to 10\%. The physician prescribed mouth washes. This was on Thursday, April 6 , and the man's condition remained about the same, face swollen, etc., until 3 o'clock in the morning of April 9 , when patient was attacked by chills, followed by a rise in temperature of two degrees. I reached the hospital at 2 p. m.

Examination.-The face was badly swollen, the swelling involving the sublingual and submaxillary glands, neck, temporal region and ear. The patient was unable to open his mouth and was suffering intense pain. The tissues were swollen to such an extent that they were very hard and it was impossible to get fluctuation. An incision had been made near the angle of the inferior maxilla internally before $I$ arrived, but nothing but blood came from the wound. I introduced a probe into the socket from whence the tooth had been extracted, but was unable to get any pus.

Trcatment.-I advised immediate operation and the patient was etherized. The mouth was forced open with a mouth prop, then with a periosteal dissector $I$ freed the muscles from the bone extending from the angle to the first molar tooth and down to the inferior border of the bone. The pus, which was deep-seated, now began to evacuate itself, until about four ounces were produced. I then curetted the tissues forming the inside of the cavity, which was as large as a hen's egg, washed it thoroughly with a weak antiseptic solution, and packed the cavity with sterilized gauze. I dressed the wound each day, and at the end of three days referred the patient back to his attending physician, under whose care he made a full recovery.

\section{GLANDULAR SWEILINGS.}

Swelling of the lymphatic glands of the neck is one of the diseases which, according to the observations of Odenthal, may be traced directly to conditions of the oral cavity. Odenthal examined 987 children and found carious teeth in 429 , in the remaining 558 no decayed teeth were present, and in 275 cases or 49 per cent. there were glandular swellings. Of the 429 children with decayed teeth, glandular swellings were present in 124 cases or 99 per cent.

In his examinations he also established a direct relation between the extent of decay and the area of involved glands. For instance, when decay was very much marked, the glands were much swollen, when decay existed on both sides glandular swelling existed on both sides, also.

\section{INYOIFEMENT OF HEART.}

Inflammation of the valves of the heart which generally follows pneumonia, can be traced without question to conditions of the oral cavity. We find the pneumococci present in cultures talien from the affected areas of the heart, after this disease has been present, and we find them in the mouth before and during the period that the person is afflicted. Therefore under these circumstances our conclusions are fair.

Many members of the medical profession including such eminent authorities as Dr. John H. Musser, of Philadelphia, consider mouth sterilization, so far as it can be done, one of the progressive steps in the treatment of pneumonia. Musser's first step in this is to place $1 \tilde{0}$ grains of calomel on the back of the tongue of the patient.

\section{RELATJON OF MOUTH INFECTIONS TO PULMONAIY} TUBERCULOSIS.

Leydon, Jaffe and James Israel demonstrated by their experiments that tuberculosis of the lungs may be traced directly to micro-organisms found in the mouth. Leyden and Jaffe in postmorten examinations of the lungs of an individual affected by putrid bronchitis or gangrene of the lungs showed that the micro-organisms were identical with those found in the mouth. Israel 
also found a small irregular body in the lung of a patient affected by primary actinomycotic infections, which on examination proved to be a salivary calculus containing bacteria which had been inspired into the lung, causing death.

Miller reports the case of a woman of 50, who was afflicted with a long-standing insufficiency of the aorta, but otherwise in good health and enjoying a vigorous digestion. After a heavy sorrow she began to lose her appetite and complained of cardialgia which set in after every meal. She was put on slight liquid nourishment, and finally was compelled to restrict herself to tea. Repeated course of treatment with Carlsbad water brought but temporary relief. Mouth examination revealed swelling and slight ulceration of the gums; besides this, the tongue was heavily coated and the posterior wall of the pharynx somewhat inflamed. Frequent syringing of the oral cavity and pharynx and also with the ioclin-myrrh tincture produced a cure in a few days, but if the patient neglected to cleanse her mouth but a single day, the old trouble would return. Miller has watched the relation between unclean mouths with swollen and inflamed gums and disorders of the stomach for many years.

$A$ case has been reported in which a man of 50 who had always been well, suddenly became troubled by inflation of the stomach and swelling of the liver. A cure was effected by the removal of an artificial upper denture which had not been taken from his mouth, not even to be cleaned, for a period of two years.

While I have treated similar cases, I selected these of authorities, such as the men mentioned in this paper, because of the high regard which both the dental and medical professions have for their careful and scientific investigations, and I am sure these cases will have a greater influence on your future observations than any number of cases which an embrvonic member of the dental profession like myself could report.

After listening to these statements which are all based on fact, and remembering that the scientist does not accept a fact until, after having a definite theory, he has taken every means to prove that his theory is wrong, we are bound as professional men to respect his records.

'Therefore it is perhaps within my rights as a dentist, to call the attention of the operating surgeon to mouth sterilization by himself. to the abolition of conversation and especially of talking into the wound during a laparotomy, as a measure towards the prevention of sepsis. The surgeon should also order sterilization of the patient's mouth before the anesthetic is given as a preventative measure against the patient drawing polluted saliva or particles of calculus such as I have described into the lungs, thus preventing post-operative pneumonia.

In nearly every pathologic condition there are inflammatory symptoms which may vary from simple hyperemia to complete destruction of the part, therefore while all conditions of oral pathology do not manifest themselves or terminate as those described, still we must admit that it is rather a question of their development than of their presence.

\section{DISCUSSION.}

DR. VIDA LATHaM, Chicago, said that what Dr. Rotch says about the ear is a good illustration that he sometimes pays little attention, and apparently does not try to reduce the number of bacteria. She hopes that the doctor will not eliminate all bacteria, because we need some special bacteria to take care of the mouth and to build up immunity and resistance to some diseases of the oral apparatus, as well as to aid in processes of digestion and oxidation.

Dr. M. I. Schamberg, Philadelphia, said that Dr. Power's observations have been the same as those of a number of other physicians. Dr. Schamberg stated that the more he hears such papers the more he believes that in dentistry we are often dealing with conditions in the mouth which encourage diseases, which may otherwise run a fatal course. He stated that dentists have a greater mission in the prevention of such fatal diseases than the man who takes the diseases after it has once placed the patient in a morjbund state. He declares that he feels proud of the work that the dental profession is doing, especially the Section on Stomatology, in combining with medical men to show the tremendous amount of good dentistry works in the entire human body.

Dr. J. E. Pownr said that he recognizes the fact that we need more bacteria in the mouth. He referred principally to the disease producing bacteria, and thinks of the two, we can, perhaps, get on better without these bacteria, than the class to which Dr. Latham referred.

\section{THE SURGICAL TREATMENT' OF DUODENAL ULCER.*}

WILLIAM D. HAGGARD, M.D. NASHVILLE, TENN.

'The duodenum is unique in its functions.' It acts as a vestibule for the small intestine and is a meeting ground for the acid stomach ingesta and the alkaline biliary and pancreatic secretion. The four inches above this point partakes of the character of the stomach and its ills, and is more frequently diseased than any other part of the intestine of equal length, save the rectum. Duodenal ulcer is much more serious than gastric ulcer. The coats are much thinner and the danger of erosion of large blood vessels is much greater and the likelihood of sudden death from perforation make it a very fearful malady.

Its symptoms are not so frank, and, when present, are either construed as gastric or they mimic the manifestations of gall-bladder disease confusingly. It is in the operating room that the real living pathology of the duodenum is being developed, and it was in the operations undertaken for one of the other above diseases that the true cause of the symptoms was disclosed to be duodenal and thus our knowledge is being enriched and the interesting and unsusupected frequency of duodenal ulcer, in comparison with gastric ulcer, demonstrated.

Burwinkel considered the ratio between gastric and duodenal ulcer as 12 to 1 . In 1896 there were but 9 reported operations. Out of 231 cases of gastrojejunostomy for ulcer, the Mayos had 119 males and $112 \mathrm{fe}$ males, of which 158 were gastric, 60 duodenal, 14 duodenal and gastric. The duodenum was, therefore, involved 74 times, or in nearly one-third.

It has the same etiology as gastric ulcer, viz.: injury of the mucosa ( 75 per cent. occur in the grinding or pyloric end), anemia and hyperchlorhydria. This latter factor is evidenced by the location of the duodenal ulcer nearly always above the entrance of the common duct with the alkaline discharge from liver and pancreas, and also by the occasional appearance of peptic ulcer in the jejunum after gastrojejunostomy where the long loop was used a number of inches from the alkaline influence of the bile and pancreatic outflow. Where the short or no-loop method is used this occurrence is rarely seen.

* Read in the Section on Surgery and Anatomy of the American Medical Association, at the Fifty-seventh Annual Session, June, 1906. 